\title{
Referral rates for children with acute gastroenteritis: a retrospective cohort study
}

\author{
Pien Ingrid Wolters' ${ }^{1}$, Gea Holtman ${ }^{1}$, Freek Fickweiler ${ }^{1}$, Irma Bonvanie ${ }^{1}$, \\ Anouk Weghorst ${ }^{1}$, Johan Post ${ }^{2}$, Boudewijn Kollen ${ }^{1}$, Marjolein Berger ${ }^{1 *}$ \\ ${ }^{1}$ Department of General Practice and Elderly Care Medicine, University Medical \\ Centre Groningen, University of Groningen, Groningen, The Netherlands; \\ ${ }^{2}$ Department of Out-of-Hours Service Groningen, Groningen, The Netherlands
}

\begin{abstract}
Background: Hospital admission rates are increasing for children with acute gastroenteritis. However, it is unknown whether this increase is accompanied by an increase in referral rates from GPs due to increased workloads in primary care out-of-hours $(\mathrm{OOH})$ services.
\end{abstract}

Aim: To assess trends in referral rates from primary care $\mathrm{OOH}$ services to specialist emergency care for children presenting with acute gastroenteritis.

Design \& setting: This retrospective cohort study covered a period from September 2007-September 2014. Children aged 6 months to 6 years presenting with acute gastroenteritis to a primary care $\mathrm{OOH}$ service were included.

Method: Pseudonymised data were obtained, and children were analysed overall and by age category. $\mathrm{X}^{2}$ trend tests were used to assess rates of acute gastroenteritis, referrals, face-to-face contacts, and oral rehydration therapy (ORT) prescriptions.

Results: The data included 12455 children (6517 boys), with a median age of 20.2 months (interquartile range [IQR] 11.6 to 36.0 months). Over 7 years, incidence rates of acute gastroenteritis decreased significantly, and face-to-face contact rates increased significantly (both, $P<0.01$ ). However, there was

*For correspondence: m.y. berger@umcg.nl

Twitter: @myberger4

Competing interest: The authors declare that no competing interests exist.

Received: 27 October 2019 Accepted: 25 November 2019

Published: 22 July 2020

@This article is Open Access: CC BY license (https://creativecommons.org/licenses/by/4.0/)

Author Keywords: After-hours care, gastroenteritis, pediatrics, paediatrics, primary care, referral

Copyright (C) 2020, The Authors;

DOI:10.3399/

bjgpopen20X101053 no significant trend for referral rates $(P=0.87)$ or prescription rates for ORT $(P=0.82)$. Subgroup analyses produced comparable results, although there was an increase in face-to-face contact rates for the older children.

Conclusion: Incidence rates for childhood acute gastroenteritis presenting in $\mathrm{OOH}$ services decreased and referral rates did not increase significantly. These findings may be useful as a reference for the impact of new interventions for childhood acute gastroenteritis.

\section{How this fits in}

Although it is known that hospital admission rates are increasing, the authors are not aware of research into the trends in referral rates for children with acute gastroenteritis presenting to primary care $\mathrm{OOH}$ services. The authors therefore investigated whether referral rates to specialist emergency care from a primary care $\mathrm{OOH}$ service increased over a 7-year period for children with acute gastroenteritis.

\section{Introduction}

Most children that are younger than 5 years will suffer from at least one episode of acute gastroenteritis. ${ }^{1}$ Although these episodes are generally self-limiting and uncomplicated, they can lead to severe 
dehydration, particularly in young children. ${ }^{2}$ Over the decade from 1999-2010, hospital admission rates for acute gastroenteritis increased by $31 \%$ in England. ${ }^{3}$ This increase has not been associated with increased severity, with most cases being for short-term admissions ( $<1$ day) that possibly could have been managed in primary care. ${ }^{3}$ High emergency admission rates are often thought to be inversely related to primary care quality, but, presumably, a complex interplay of factors is responsible for the observed increase in hospital admission rates. ${ }^{4}$

Primary care $\mathrm{OOH}$ services are regional centres in which multiple GPs work in shifts to cover patients outside of normal working hours. ${ }^{5}$ Patients in the Netherlands must go through triage by telephone before they are invited for face-to-face contact with a GP in the $\mathrm{OOH}$ service. Factors thought to have influenced the increase in hospital admission rates include complicated access to the $\mathrm{OOH}$ service, loss of continuity in GP care, a drive for shorter hospital stays (also leading to increased readmission rates), the impact of social media, and the expectations of parents and professionals for the treatment of a sick child. ${ }^{4}$ In addition, GPs are experiencing high workloads in $\mathrm{OOH}$ services, ${ }^{6}$ which may be due to inaccurate triage of children by telephone assistants. In turn, this may contribute to more referrals to paediatric emergency departments and consequent hospital admissions for children who could be better managed at home with ORT. ${ }^{7}$

Although trends in hospital admission rates are known, the authors are not aware of research into the trends in referral rates for children with acute gastroenteritis presenting to primary care $\mathrm{OOH}$ services. The authors therefore investigated whether referral rates to paediatric emergency care from a primary care $\mathrm{OOH}$ service increased over a 7-year period for children with acute gastroenteritis. In addition, factors potentially related to that trend were explored, focusing on rates of the incidence of acute gastroenteritis, face-to-face contacts, and ORT prescriptions.

\section{Method}

\section{Study design}

This retrospective cohort study was performed using information obtained between September 2007-September 2014. Data for children aged 6 months to 6 years were obtained from the electronic database of a primary care $\mathrm{OOH}$ service. The primary outcome was the referral rate from this service to secondary care.

\section{Setting and triage procedure}

Pseudonymised data were obtained from the electronic database of a primary care $\mathrm{OOH}$ service that included 290 collaborating GPs providing care for approximately 650000 residents in the north of the Netherlands. ${ }^{8}$ Triage was initially performed over the telephone by trained assistants who assessed the urgency of a consultation based on the guidelines of the Dutch College of General Practitioners. They were then able to offer advice over the telephone — including advice to administer ORT — or make an appointment for face-to-face contact with a GP. If the patient was seen by a GP in a faceto-face contact, the GP decided if referral was necessary or if the patient could be managed at home. The assistant and GP record their findings in the patient's medical record, which contains information on the contact date, demographics, symptoms, physical examination, additional testing, diagnosis, prescriptions, and referrals.

\section{Study population and contact selection}

The study included children aged 6 months to 6 years who were diagnosed with acute gastroenteritis and seen in the $\mathrm{OOH}$ service during the study period. First, all contacts of children aged 6 months to 6 years were selected, and their medical records were extracted and saved in a database. All patient information was pseudonymised by the $\mathrm{OOH}$ service. Second, a computer search was performed to select all contacts with the words 'diarrhoea' and/or 'vomiting' (or synonyms of these words) in the history record. The results were checked for false negatives by randomly extracting $10 \%$ of all $\mathrm{OOH}$ service contacts over the study period $(n=5000)$ and hand checking if any children with diarrhoea and/or vomiting had been missed. The computerised search was then adapted, and the false negative screening was repeated until no eligible contacts were missed. Three researchers (two medical students and a GP) also hand searched all contacts in which the child presented with diarrhoea and/or vomiting to exclude those with chronic diarrhoea (that is to say, those with symptoms for $\geq 2$ weeks). 
The study defined a diagnosis of acute gastroenteritis as follows: 1) a registered diagnosis of 'gastroenteritis', or synonyms; or 2) a registered diagnosis of 'viral infection' or 'vomiting' if diarrhoea was a presenting symptom; or 3 ) if no diagnosis was recorded, but diarrhoea or vomiting was the presenting symptom and other plausible causes were not mentioned. Contact selection was performed by three researchers, and any uncertainties were discussed with an expert panel (two GPs). If children contacted more than once within two weeks, it was counted as one episode. If children contacted more than once, but with an intervening period of more than 2 weeks, this was counted as separate episodes.

\section{Data extraction}

The following data were extracted by three researchers using a structured form: contact date, contact type (telephone, face-to-face), age, symptoms and signs, referral, and any medication or ORT prescribed (or self-prescribed). Before starting full data extraction, a pilot was performed to determine the level of agreement between researchers in the extracted data (Cronbach's alpha, 20.87). After a consensus meeting, agreement was retested for a random sample of $10 \%$ of all of the included contacts (Cronbach alpha, $\geq 0.85$ ). Thus, there was a good level of agreement in the information extracted between researchers.

\section{Outcomes}

The primary outcome was the referral rate, with the total number of contacts per year as the denominator. Secondary outcomes were to analyse the incidence rate of acute gastroenteritis, the rate of each contact type (for example, face-to-face or telephone), and the rate of ORT prescriptions.

\section{Statistical analysis}

Descriptive data are reported as medians and IQRs, or as numbers and percentages. Trends were evaluated for all primary and secondary outcomes. In addition, subgroup analyses were performed for age categories of 6 to 12 months and 1 to 6 years. All trend analyses were conducted using the $\mathrm{X}^{2}$ test (two-sided), and were considered significant if $P<0.05$. Data were analysed using IBM SPSS (version 25.0).

\section{Results}

\section{Patient characteristics}

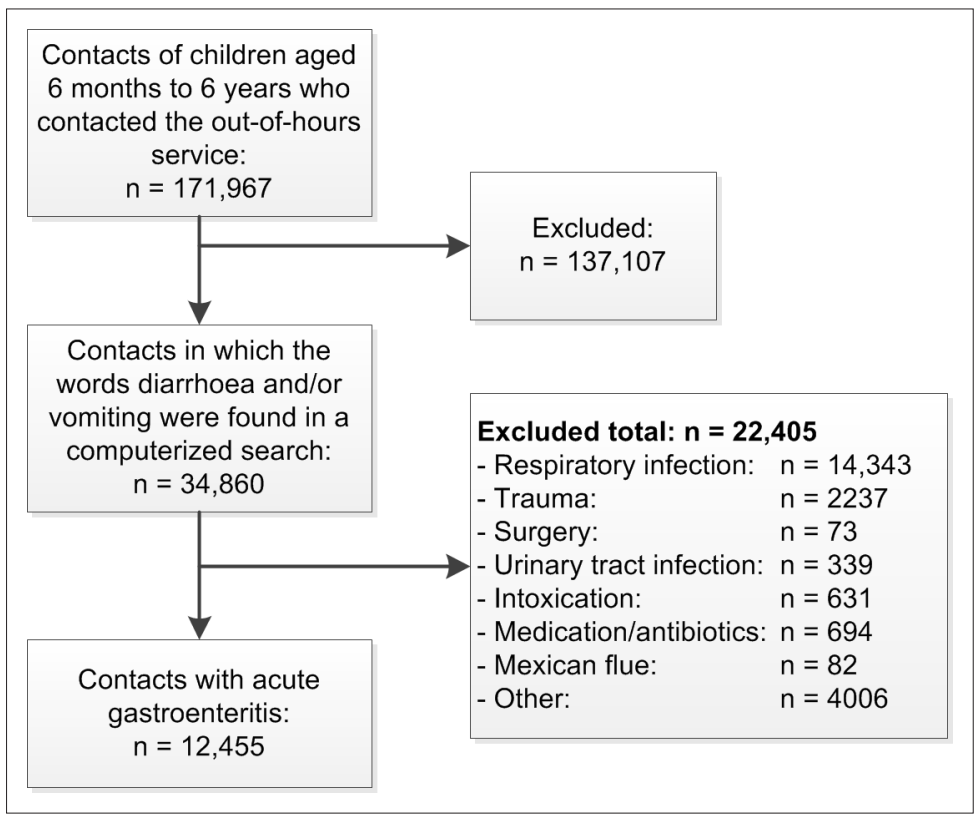

Figure 1 Flowchart of patient selection 
Table 1 Characteristics of Dutch children aged 6 months to 6 years with acute gastroenteritis who were seen in a primary care out-of-hours service (2007-2014)

\begin{tabular}{lcc}
\hline Characteristics & Total $(n=12455)$ & Referred children $(\boldsymbol{n}=1036)$ \\
\hline Male sex, $n(\%)$ & $6517(52.3)$ & $556(53.7)$ \\
\hline Median age, months (IQR) & $20.2(11.6$ to 36.0) & $18.0(12.0$ to 32.0) \\
\hline Age categories & $3229(25.9)$ & $249(24.0)$ \\
\hline 6 months to $<1$ year, $n(\%)$ & $9226(74.1)$ & $787(76.0)$ \\
\hline 1 to 6 years, $n(\%)$ & & $106(10.2)^{b}$ \\
\hline Presenting symptoms & $2678(21.5)^{\mathrm{a}}$ & $214(20.7)^{\mathrm{d}}$ \\
\hline Diarrhoea only, $n(\%)$ & $3934(31.6)^{\mathrm{c}}$ & $716(69.1)$ \\
\hline Vomiting only, $n(\%)$ & $5843(46.9)$ & \\
\hline Diarrhoea and vomiting, $n(\%)$ & & \\
\hline
\end{tabular}

${ }^{a}$ No information about vomiting in patient record $(n=1621)$.

${ }^{\text {b} N o ~ i n f o r m a t i o n ~ a b o u t ~ v o m i t i n g ~ i n ~ p a t i e n t ~ r e c o r d ~}(n=73)$.

'No information about diarrhoea in patient record $(n=2006)$

No information about diarrhoea in patient record $(n=112)$.

$\mathrm{IQR}=$ interquartile range.

In total, 171967 contacts with the $\mathrm{OOH}$ service were recorded for children aged 6 months to 6 years during the study period. Among these, 34860 were for diarrhoea and/or vomiting, and a subset of 12455 (9432 children) were diagnosed with acute gastroenteritis (Figure 1). For those with acute gastroenteritis, multiple contacts were recorded in 3023 cases (specifically, two times for 1613 children, three times for 396 children, four times for 112 children, five times for 45 children, six times for 13 children, seven times for five children, and eight times for one child).

The median age was 20.2 months (IQR 11.6 to 36.0), and boys accounted for 6517 contacts (52.3\%). Regarding presentation, 2678 (21.5\%) contacts had only diarrhoea, 3934 (31.6\%) had only vomiting, and $5843(46.9 \%)$ had both diarrhoea and vomiting. In total 9777 (78.5\%) contacts presented with vomiting and 6614 (53.1\%) with diarrhoea (Table 1). For the 1036 contacts (8.3\%) referred with acute gastroenteritis, age and sex were comparable to those in the overall cohort, but a higher proportion had both diarrhoea and vomiting.

\section{Trend analyses}

Table 2 presents the results of the trend analyses overall and for the two age subgroups over the 7 -year study period. In both the overall and subgroup analyses, no significant increase in the trend for referral rates was found (overall median $8.1 \%$ ). However, there was an increasing trend in face-toface contact rates for all children with acute gastroenteritis $(P<0.01)$. Subgroup analyses confirmed that this increasing trend was only statistically significant for children aged $1-6$ years $(P<0.01)$. ORT prescription rates did not change significantly $(P=0.82)$. Finally, there was a significantly decreasing trend in the incidence rate of acute gastroenteritis presenting to the $\mathrm{OOH}$ service in both the overall and the subgroup analyses $(P<0.01)$.

\section{Discussion}

\section{Summary}

This study gives important insights into referral rates for childhood acute gastroenteritis from a primary care $\mathrm{OOH}$ service to paediatric specialist care between 2007 and 2014, a period during which there was no change in guidelines. Incidence rates for childhood acute gastroenteritis decreased and this study could not show a trend in referral rates in both the overall and subgroup analyses. The median referral rate was $8.1 \%$. The study found a statistically significant increasing trend in face-toface contact rates. This was mainly due to a significant increasing trend in face-to-face contact rates in 


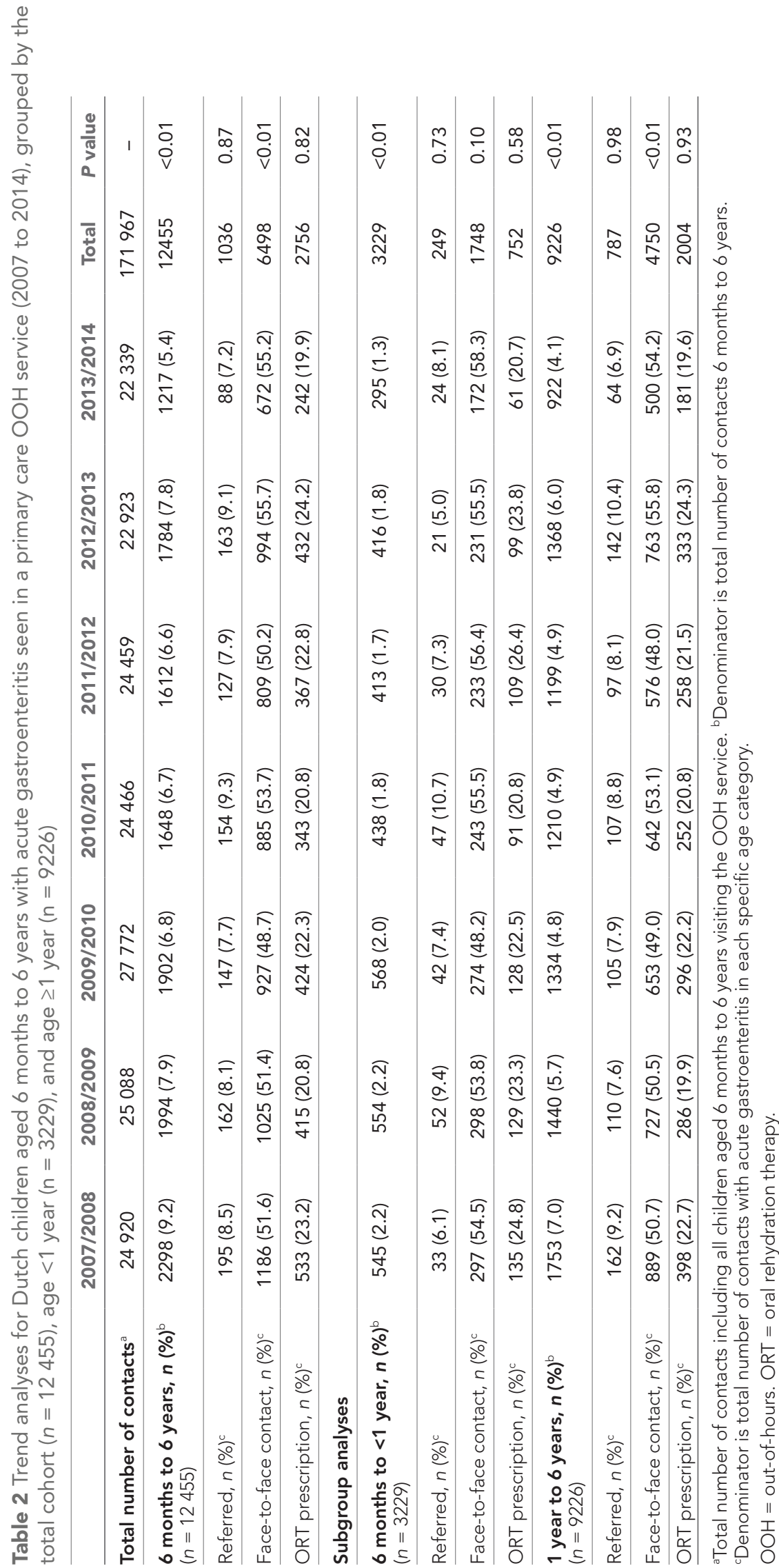


children aged 1-6 years. Referral was more likely for children reporting both diarrhoea and vomiting, and almost one in five children received advice or a prescription for ORT.

\section{Strengths and limitations}

The main strength was the inclusion of a relatively large number of patient contacts. Data were then obtained in a structured manner with good reliability among the raters and discussion of doubtful contacts. Missing data were also minimised because Dutch law (The Medical Treatment Agreement Act) requires that information on referrals and prescriptions be recorded. Moreover, when the authors screened a random sample for false negatives, they confirmed that few children with diarrhoea and/ or vomiting were missed by the computerised selection method.

Some limitations do need to be considered, such as the decision to include only those aged 6 months to 6 years, and to perform subgroup analysis at a cut-off of 1 year. The overall age range was chosen because it corresponded to the peak incidence of acute gastroenteritis ${ }^{10}$ and the group that most often contacts primary care $\mathrm{OOH}$ services. ${ }^{5}$ Younger children were excluded because they are at increased risk of dehydration, meaning that any referral decisions may only reflect age. ${ }^{11}$ In the subgroup analyses, age groups were predefined based on their assumed risk for a complicated course. However, this age cut-off was arbitrary, and it may have been preferable to use the 2 year cutoff advised in the 2014 revision of Dutch guidelines. ${ }^{10}$ Furthermore, multiple contacts were recorded in 3023 cases, which might have influenced the magnitude of the referral rate.

The health care system in the Netherlands is comparable to those in Denmark, Sweden, the UK, Australia, and New Zealand, which are based on the GP serving as a gatekeeper to further care. ${ }^{12}$ However, watchful waiting is a common strategy in the Netherlands, with emphasis on telephone advice and relatively few people getting face-to-face contact with the GP. ${ }^{13}$ For example, $5 \%$ and $22 \%$ of community cases visit their GP because of acute gastroenteritis in the Netherlands and New Zealand, respectively. ${ }^{14}$ Therefore, trends in referral rates in the Netherlands could also differ from those in other countries.

\section{Comparison with existing literature}

It was notable that there was no statistically significant increase in referral rates from the $\mathrm{OOH}$ service to the emergency department, which ran counter to the authors' expectation based on a previous report on increasing hospital admission rates. ${ }^{3}$ The findings may indicate that parents attend the paediatric emergency department directly, possibly because of easier access to advice without the need for telephone triage. ${ }^{4}$ This could account for the increase in hospital admission rates of children that could be managed in primary care, despite referral rates from $\mathrm{OOH}$ services remaining stable. However, this does not seem a plausible explanation. Given that prognosis was worse among those self-referred with fever, parents appear to be capable of accurately evaluating the severity of illness and need for emergency paediatric care. ${ }^{15}$

The increasing trend in face-to-face contact rates for acute gastroenteritis in children was consistent with the findings of a Dutch study showing a similar increase in face-to-face contact rates to $\mathrm{OOH}$ services for other problems between 2009 and 2016. ${ }^{6}$ This may indicate a change in telephone triage practices at $\mathrm{OOH}$ services. In the Netherlands, most $\mathrm{OOH}$ services use a validated standard for triage to increase its efficiency and patient safety. ${ }^{9}$ However, unknown patients, anxious parents, high work pressures, ${ }^{16}$ and differing views of disease and illness can make triage challenging. Furthermore, it has been shown that telephone triage may be especially suboptimal for children with gastrointestinal complaints. ${ }^{17}$ These challenges may be associated with the increase in face-to-face contact rates for children with benign prognoses. This in turn, may contribute to the high work pressure experienced by GPs in these services, even while the absolute number of children presenting with acute gastroenteritis decreases. ${ }^{6}$

Subgroup analyses showed that the increase in face-to-face contact rates was only significant for children aged 1-6 years, in whom the risks of complications were low to moderate. It is unlikely that risk actually increased over time to justify this change, indicating that more children with a benign prognosis were allowed through to face-to-face contacts. Indeed, despite the increase in face-to-face contact rates, secondary triage by GPs did not result in a corresponding increase in referral rates. The likelihood of referral was increased if the child had an increased risk of dehydration, with referral rates 
being highest for those presenting with both vomiting and diarrhoea. This finding indicates that the quality of GP triage remained appropriate.

ORT is the recommended first line treatment for children at risk of dehydration or with mild, moderate, or severe dehydration, with proven efficacy. ${ }^{2}$ In the present study, the change in ORT prescribing rates was statistically insignificant, with approximately $22 \%$ receiving advice or a prescription, and non-prescribing justified by the presence of vomiting in about $80 \%$ of patients. ${ }^{7}$ This is consistent with the justifiable fear that vomiting will hamper the intake of ORT and may affect compliance. In referred children, ondansetron is an effective antiemetic, which could increase ORT uptake and compliance. ${ }^{18}$ As such, this medication may have a role in primary care, with the potential to prevent referrals to secondary care and manage patients in primary care.

The incidence rate of acute gastroenteritis in children almost halved during the study period (Table 2). However, this finding should be interpreted with caution because the annual incidence fluctuates widely for a range of reasons, with each of these having the potential to explain the observed variations. ${ }^{19}$ Moreover, only the first and last years of this study showed markedly different incidence rates, with relative stability observed in the intervening period. An explanation for the lower incidence and referral rates for acute gastroenteritis in 2013-2014 could be the lower reported incidence of rotavirus infections in that year. ${ }^{20}$ Given that rotavirus is known to be associated with a particularly complicated course, ${ }^{21}$ a lower incidence could be associated with fewer contacts and referrals.

\section{Implications for research and practice}

These results showed that the trend in referral rates to secondary care is not significant. There are five aspects in the management of children with acute gastroenteritis that could potentially affect these rates in the future. First, the introduction of point-of-care tests for pathogens of acute gastroenteritis may affect management. Triaging children with gastrointestinal complaints based on their clinical signs and symptoms is challenging. It is therefore unsurprising that GPs have difficulties in distinguishing between children who will have uncomplicated courses and those who will have complicated courses requiring referral. Current guidelines do not recommend stool microbiological investigation for acute gastroenteritis in children. ${ }^{10,11}$ Research could therefore evaluate if specific pathogens are associated with a more severe course of acute gastroenteritis, and if demonstrated, should assess the added value of point-of-care tests in daily practice.

Second, rotavirus is the most common pathogen among children presenting with acute gastroenteritis in primary care, ${ }^{10}$ yet children had not been vaccinated against it during the study period. Implementing this vaccination in the future will influence the risk of a complicated course in children with acute gastroenteritis. This will influence the need for referral.

Third, although referral rates remained constant at a median of $8.1 \%$, the percentage that was subsequently admitted to hospital was unknown. It would be interesting to know whether treating vomiting specifically could facilitate greater ORT intake in primary care, and thereby decrease referral rates. Ondansetron is often used with good efficacy as an antiemetic, and to increase ORT uptake and compliance in paediatrics. ${ }^{18}$ For now, ondansetron has only been shown to have benefit in hospital settings, at the more severe end of the spectrum, especially in children who are deemed unsuitable for discharge from emergency department. It might be argued that ondansetron may not be warranted, safe, or cost-efficient in children presenting to primary care. Concerns about diarrhoea, prolongation of QT-interval on electrocardiogram, and prescribing for minimal clinical benefit may challenge uptake in primary care. A study addressing the impact of ondansetron in primary care, focusing on children with acute gastroenteritis and prominent vomiting, is therefore highly needed.

A fourth management aspect is that not enough is known about the adherence to prescriptions for ORT. The presence of vomiting in around $80 \%$ of contacts in this study could result in poor compliance with ORT, or to GPs fearing poor compliance. Further research into ORT adherence, including qualitative research into the barriers to adherence, is therefore warranted.

Finally, the reasons for the increase in face-to-face contact by older children requires further research. Important questions in this research will include the reasons for parents contacting $\mathrm{OOH}$ services, the validity of telephone triage, and the availability of adequate and appropriate information about when parents should contact the $\mathrm{OOH}$ service.

In a 7-year period from 2007 to 2014, incidence rates for childhood acute gastroenteritis presenting at $\mathrm{OOH}$ services decreased, and referral rates remained stable. These findings may be useful as a 
reference against which the impact of new interventions for childhood acute gastroenteritis can be measured.

\section{Funding}

No external funding was received.

\section{Ethical approval}

The Medical Ethics Review Committee of the University Medical Centre Groningen approved this study and confirmed that it did not require further ethical approval according to the Dutch Medical Research Involving Human Subject Act (WMO).

\section{Provenance}

Freely submitted; externally peer reviewed

\section{Acknowledgements}

The authors would like to thank the out-of-hours service at Groningen for providing the patient data used in this study. They also thank Dr Robert Sykes (www.doctored.org.uk) for providing editorial services.

\section{References}

1. Parashar UD, Hummelman EG, Bresee JS, et al. Global illness and deaths caused by rotavirus disease in children. Emerg Infect Dis 2003; 9(5): 565-572. DOI: https://doi.org/10.3201/eid0905.020562

2. Guarino A, Ashkenazi S, Gendrel D, et al. European Society for pediatric gastroenterology, hepatology, and utrition/European society for pediatric infectious diseases evidence-based guidelines for the management of acute gastroenteritis in children in Europe: update 2014. J Pediatr Gastroenterol Nutr 2014; 59(1): 132-152. DOI: https:// doi.org/10.1097/MPG.0000000000000375

3. Gill PJ, Goldacre MJ, Mant D, et al. Increase in emergency admissions to hospital for children aged under 15 in England, 1999-2010: national database analysis. Arch Dis Child 2013; 98(5): 328-334. DOI: https://doi.org/10. 1136/archdischild-2012-302383

4. Neill S, Roland D, Thompson $M$, et al. Why are acute admissions to hospital of children under 5 years of age increasing in the UK? Arch Dis Child 2018; 103(10): 917-919. DOI: https://doi.org/10.1136/archdischild-2017313958

5. Jansen T, Ramerman L, Verheij R. [Care by the doctor. Nivel Care registrations first line: annual figures 2018 and trend figures 2014-2018] Zorg op de huisartsenpost. Nivel Zorgregistraties Eerste Lijn: jaarcijfers 2018 en trendcijfers 2014-2018. (in Dutch). Utrecht: Nivel; 2020. https://nivel.nl/nl/publicatie/zorg-op-de-huisartsenpostnivel-zorgregistraties-eerste-lijn-jaarcijfers-2018-en

6. InEen. [Benchmark bulletin general practitioner posts 2016] Benchmarkbulletin huisartsenposten 2016 (in Dutch). Utrecht; 2016.

7. Chow CM, Leung AK, Hon KL. Acute gastroenteritis: from guidelines to real life. Clin Exp Gastroenterol 2010; 3(1): 97-112. DOI: https://doi.org/10.2147/CEG.S6554

8. Scholten I. [Annual report doctor service 2016] Jaarverslag doktersdienst 2016 (in Dutch). Groningen; 2016.

9. Domus Medica. [Vision and working method NTS] Visie en werkwijze NTS (in Dutch). Utrecht; 2017.

10. Belo J, Bos M, Brühl P, et al. [NHG standard acute diarrhoea 2014] NHG-Standaard acute diarree 2014 (in Dutch) 2014; 57(9): 462-471.

11. NICE. Diarrhoea and vomiting caused by gastroenteritis in under 5S: diagnosis and management. 1-38. 2009; https://www.nice.org.uk/guidance/CG84 (accessed 30 May 2020).

12. Busse R, Dixon A, Healy J, et al Health care systems in eight countries: trends and challenges European Observatory. London: European Observatory on Health Care Systems; 2002. https://www.mig.tu-berlin.de/ fileadmin/a38331600/2002.publications/2002.busse_ObservatoryWanless.pdf

13. Koopmans MPG, Kortbeek LM, van Duynhoven Y. [Acute gastroenteritis: insight into the incidence, causes and diagnostics by population research] Acute gastro-enteritis: inzicht in incidentie, oorzaken en diagnostiek door populatieonderzoek (in Dutch). Tijdschr Infect 2008; 3(1): 8-16.

14. Lake R, Adlam B, Perera S. Acute Gastrointestinal Illness (AGI) Study: Final study report. Christchurch; 2009.

15. van lerland $Y$, Seiger $N$, van Veen $M$, et al. Self-referral and serious illness in children with fever. Pediatrics 2012; 129(3): e643-e651. DOI: https://doi.org/10.1542/peds.2011-1952

16. van der Linden MC, Meester BEAM, van der Linden N. Emergency department crowding affects triage processes. Int Emerg Nurs 2016; 29: 27-31. DOI: https://doi.org/10.1016/j.ienj.2016.02.003

17. Scarfone RJ, Luberti AA, Mistry RD. Children referred to an emergency department by an after-hours call center: complaint-specific analysis. Pediatr Emerg Care 2004; 20(8): 507-513. DOI: https://doi.org/10.1097/01.pec. 0000136066.99456 .41 
18. Tomasik E, Ziółkowska E, Kołodziej M, Szajewska H. Systematic review with meta-analysis: ondansetron for vomiting in children with acute gastroenteritis. Aliment Pharmacol Ther 2016; 44(5): 438-446. DOI: https://doi.org/ 10.1111/apt.13728

19. Grassly NC, Fraser C. Seasonal infectious disease epidemiology. Proc Biol Sci 2006; 273(1600): 2541-2550. DOI: https://doi.org/10.1098/rspb.2006.3604

20. Hahné S, Hooiveld M, Vennema H, et al. Exceptionally low rotavirus incidence in the Netherlands in $2013 / 14$ in the absence of rotavirus vaccination. Euro Surveill 2014; 19(43): 2094530 Oct 2014. DOI: https://doi.org/10.2807/15607917.ES2014.19.43.20945

21. Wiegering V, Kaiser J, Tappe D, et al. Gastroenteritis in childhood: a retrospective study of 650 hospitalized pediatric patients. Int J Infect Dis 2011; 15(6): e401-e407. DOI: https://doi.org/10.1016/j.ijid.2011.02.006 